\title{
Docking Studies of Pyrimidine Analogues on Geranyl Transferase
}

Ethiraj KR ${ }^{1}$, Vino $\mathbf{S}^{2}$, Nawas Khan $\mathrm{F}^{1}$ and Sajitha Lulu $\mathbf{S}^{2 *}$

${ }^{1}$ School of Advanced Sciences, VIT University, Vellore, Tamilnadu-632014, India

${ }^{2}$ School of Biosciences and Technology, VIT University, Vellore, Tamilnadu-632014, India

\begin{abstract}
Rab geranylgeranyltransferase catalyzes the attachment of geranylgeranyl isoprenoids to Rab guanine triphosphatases, which are the key regulators in vesicular transport. The identification of the binding interactions between the RabGGTase and pyrimidine analogues can facilitate the anti-cancer drug design studies. In this study, GLIDE software was used to dock a series of pyrimidine analogues to RabGGTase. Good agreement was observed between the top experimental inhibitors and the top ranked docking results, and key interactions between the ligands and receptor were identified.
\end{abstract}

Keywords: RabGGTase; Docking; Prenyltransferases; OPLS-AA force field; Pyrimidine; QuickPro; GLIDE

\section{Introduction}

Rab proteins are small GTPases involved in the regulation of vesicular traffic [1]. To be functional, they have to undergo a post-translational modification in which one or two isoprenoid geranylgeranyl groups are attached to their C termini [2]. Rab geranylgeranyltransferase (RabGGTase or GGTase type II) catalyzes this reaction. RabGGTase belongs to a family of prenyltransferases that includes farnesyltransferase (FTase) and geranylgeranyltransferase type I. These enzymes are highly conserved in sequence and structure and possess a single active site [3].

Pyrimidines are an important class of heterocyclic compounds, which possess a wide range of biological activities such as anticancer [4,5], antibacterial [6], anti-inflammatory [7], antiviral [8], antitubercular [9], antihypertensive [10] and anticonvulsant [11] activities. The presence of $4-\mathrm{OCH}_{3}$ and $2-\mathrm{NO}_{2}$ and $3-\mathrm{NO}_{2}$ substitutions on pyrimidines shows significant increase in antitubercular activity [12]. The bromo substituted, 2-hydroxy phenyl substituted pyrimidines also shows good antihistaminic activity [13].

To know the possibility of binding of RabGGTase protein to pyrimidine analogues is fascinating and molecular docking is frequently used to predict the binding orientation. Docking is used to bring out new ligands for biological targets with a known 3D structure [14]. Docking studies of the compounds were performed using GLIDE (Schrödinger, LLC, Portland) $[15,16]$.

\section{Materials and Methods}

\section{Ligand structure preparation}

The structures of six pyrimidine analogues, a) 4-(2-fluoro-4methoxyphenyl)-6-(2,3, trimethoxyphenyl) pyrimidin-2-amine, b) 4-(2-fluoro-4-methoxyphenyl)-6-(2,4,6-trimethoxyphenyl) pyrimidin2-amine, c) 4-(2-fluoro-4-methoxyphenyl)-6-(2, 4-dimethoxyphenyl) pyrimidin-2-amine, d) 4-(2-fluoro-4-methoxyphenyl)-6(2,4,5-trimethoxyphenyl) pyrimidin-2-amine, e) 4-(2-fluoro-4methoxyphenyl)-6-(3,4,5-trimethoxyphenyl) pyrimidin-2-amine, f) 4-(2-fluoro-4-methoxyphenyl)-6-(3,5-dimethoxyphenyl) pyrimidin2 -amine (Figure 1) were constructed using the fragment dictionary of Maestro 7.5 (Schrodinger, LLC) using the Optimized Potentials for Liquid Simulations-All Atom (OPLS-AA) force field [17] with the steepest descent followed by truncated Newton conjugate gradient protocol. Partial atomic charges were computed using the OPLS-AA force field. The ligand molecules and their methoxy substitution are shown in table 1 .

\section{Protein structure preparation}

The X-ray crystal structure of protein geranyl transferase in complex with a peptidomimetic inhibitor (PDB ID: 3HXB) obtained from the Research Collaboratory for Structural Bioinformatics (RCSB) Protein Data Bank (Figure 2) was used in this study. Water molecules of crystallization were removed from the complex and the protein was optimized for docking using the protein preparation and refinement utility provided by Schrödinger LLC. Partial atomic charges were assigned according to the OPLS-AA force field.

\section{Docking protocol}

All docking calculations were performed using the "Extra Precision" (XP) mode of GLIDE program. The binding site, for which the various energy grids were calculated and stored, is defined in terms of two concentric cubes: the bounding box, which must contain the center of any acceptable ligand pose, and the enclosing box, which must contain all ligand atoms of an acceptable pose, with a Root Mean Square Deviation (RMSD) of less than $0.5 \AA$ and a maximum atomic displacement of less than $1.3 \AA$ were eliminated as redundant in order to increase diversity in the retained ligand poses. The scale factor for van der Waals radii was applied to those atoms with absolute partial charges less than or equal to 0.15 (scale factor of 0.8 ) and 0.25 (scale factor of 1.0) electrons for ligand and protein, respectively. The max keep variable which sets the maximum number of poses generated during the initial phase of the docking calculation were set to 5000 and the keep best variable which sets the number of poses per ligand that enters the energy minimization was set to 1000. Energy minimization protocol includes dielectric constant of 4.0 and 1000 steps of conjugate gradient. Upon completion of each docking calculation, at most 100 poses per ligand were generated. The best docked structure was chosen using a GLIDE score (Gscore) function. Another scoring function used by GLIDE is E-model, which itself derived from a combination of the Gscore, Coulombic, van der Waals and the strain energy of the ligand.

*Corresponding author: Sajitha Lulu S, Assistant Professor, School of Biosciences and Technology, VIT University, Vellore, Tamil Nadu-632014, India, Tel: +91 9944807641; E-mail: ssajithalulu@vit.ac.in

Received April 29, 2011; Published January 15, 2013

Citation: Ethiraj KR, Vino S, Nawas Khan F, Sajitha Lulu S (2013) Docking Studies of Pyrimidine Analogues on Geranyl Transferase. 2: 610 doi:10.4172 scientificreports.610

Copyright: @ 2013 Ethiraj KR, et al. This is an open-access article distributed under the terms of the Creative Commons Attribution License, which permits unrestricted use, distribution, and reproduction in any medium, provided the original author and source are credited. 
Citation: Ethiraj KR, Vino S, Nawas Khan F, Sajitha Lulu S (2013) Docking Studies of Pyrimidine Analogues on Geranyl Transferase. 2: 610 doi:10.4172/scientificreports.610
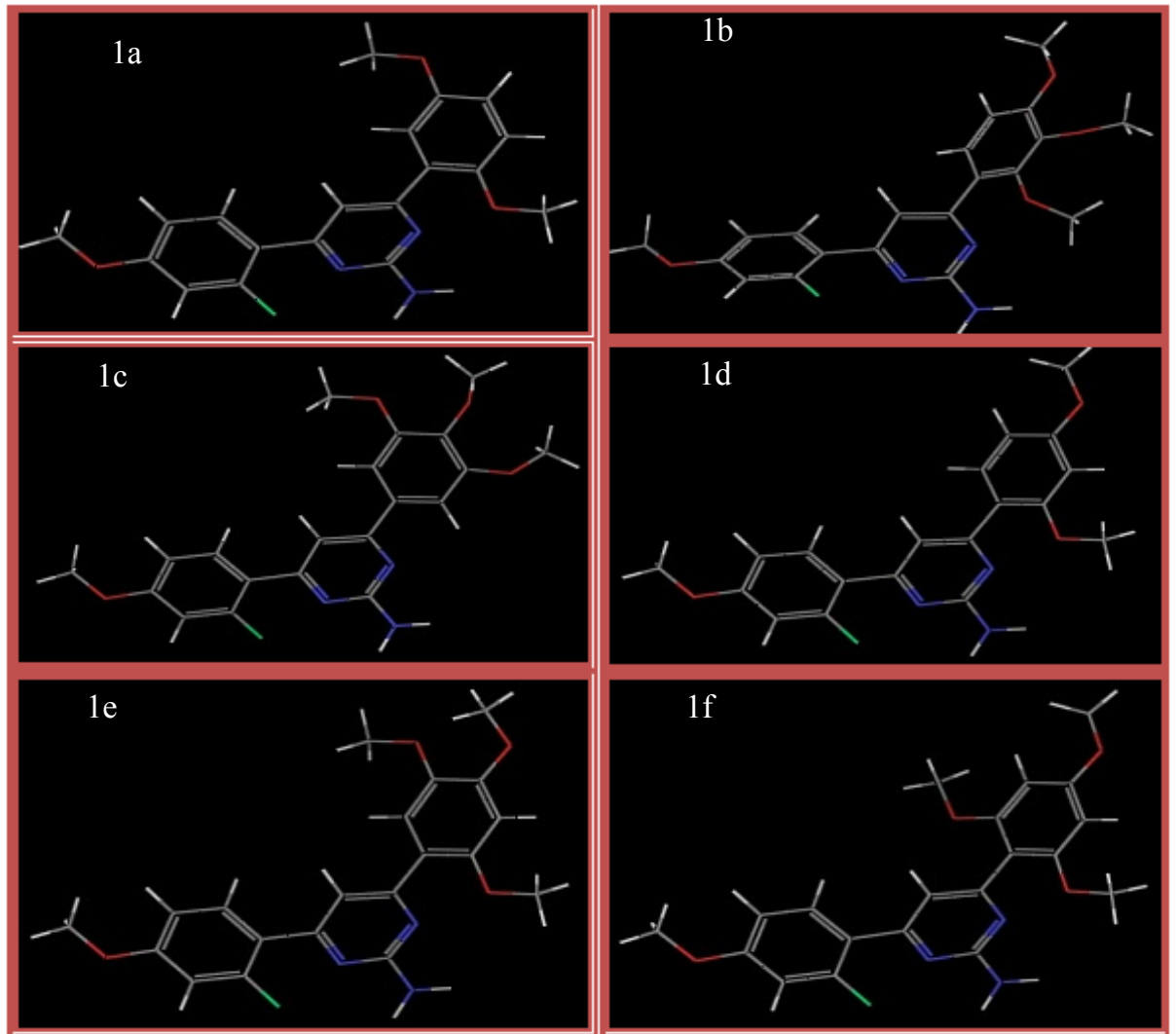

Figure 1: Structures of different ligands. (a) Ligand 1: 4-(2-fluoro-4-methoxyphenyl)-6-(3,4,5-trimethoxyphenyl) pyrimidin-2-amine, (b) Ligand 2: 4-(2-fluoro-4-methoxyphenyl)-6-(2,4,6-trimethoxyphenyl) pyrimidin-2-amine, (c) Ligand 3: 4-(2-fluoro-4-methoxyphenyl)-6-(2,3-trimethoxyphenyl) pyrimidin-2-amine, (d) Ligand 4: 4-(2-fluoro-4-methoxyphenyl)-6-(2,4, 5-trimethoxyphenyl) pyrimidin-2-amine, (e) Ligand 5: 4-(2-fluoro-4methoxyphenyl)-6-(2,4-dimethoxyphenyl) pyrimidin-2-amine, (f) Ligand 6: 4-(2-fluoro-4-methoxyphenyl)-6-(3,5-dimethoxyphenyl) pyrimidin-2-amine.

\begin{tabular}{|c|c|c|c|c|c|c|}
\hline S.No & Ligand & R1 & $\mathbf{R} 2$ & R3 & R4 & R5 \\
\hline 1 & Ligand 1 & $\mathrm{OCH}_{3}$ & $\mathrm{OCH}_{3}$ & $\mathrm{OCH}_{3}$ & $\mathrm{H}$ & $\mathrm{H}$ \\
\hline 2 & Ligand 2 & $\mathrm{OCH}_{3}$ & $\mathrm{H}$ & $\mathrm{OCH}_{3}$ & $\mathrm{H}$ & $\mathrm{OCH}_{3}$ \\
\hline 3 & Ligand 3 & $\mathrm{OCH}_{3}$ & $\mathrm{H}$ & $\mathrm{OCH}_{3}$ & $\mathrm{H}$ & $\mathrm{H}$ \\
\hline 4 & Ligand 4 & $\mathrm{OCH}_{3}$ & $\mathrm{H}$ & $\mathrm{OCH}_{3}$ & $\mathrm{OCH}_{3}$ & $\mathrm{H}$ \\
\hline 5 & Ligand 5 & $\mathrm{H}$ & $\mathrm{OCH}_{3}$ & $\mathrm{OCH}_{3}$ & $\mathrm{OCH}_{3}$ & $\mathrm{H}$ \\
\hline 6 & Ligand 6 & $\mathrm{H}$ & $\mathrm{OCH}_{3}$ & $\mathrm{H}$ & $\mathrm{OCH}_{3}$ & $\mathrm{H}$ \\
\hline
\end{tabular}

Table 1: Methoxy substitution on pyrimidines.

\section{QikProp analysis}

QikProp efficiently evaluates pharmaceutically relevant properties for over half a million compounds per hour, making it an indispensable lead generation and lead optimization tool. Accurate prediction of Absorption, Distribution, Metabolism, Elimination (ADME) properties prior to expensive experimental procedures, such as High Throughput Screening (HTS), can eliminate unnecessary testing on compounds that will ultimately fail; ADME prediction can also be used to focus lead optimization efforts to enhance the desired properties of a given compound $[15,16]$.

\section{SiteMap analysis}

SiteMap proven algorithm for binding site identification and evaluation can help researchers to locate binding sites with a high degree of confidence and predict the druggability of those sites. Beyond lead discovery, SiteMap assists in lead optimization by providing insight into ligand-receptor interactions so as to suggest effective strategies to modify lead compounds to enhance receptor complementarity [17].

\section{Results and Discussion Results from QikProp}

The ADME properties of the designed ligands were predicted using QikProp. The compounds prepared were subjected to druglikeness filter. The criteria of the filter includes molecular weight 160 480 , number of heavy atoms 20-70, lipophilicity 40-130, Number of hydrogen bond donors 4-7, Number of hydrogen bond acceptors 8-12. All the designed ligands conformed to the above mentioned criteria and they were evaluated for docking using GLIDE software.

\section{Results from SiteMap}

The active site on the protein was predicted by SiteMap (Figure 3). The protein consisted of five active sites. The yellow, blue and red meshes represent the hydrophobic map, hydrogen bond donor, and hydrogen bond acceptor respectively.

\section{Receptor grid generation}

GLIDE receptor grid was generated to determine the size of the 
Citation: Ethiraj KR, Vino S, Nawas Khan F, Sajitha Lulu S (2013) Docking Studies of Pyrimidine Analogues on Geranyl Transferase. 2: 610 doi:10.4172/scientificreports. 610

Page 3 of 5

active site (Figure 4). The most probable orientation of the ligands in the binding pocket is identified and a scoring function is used to quantify the strength of the interaction a molecule can make in a particular orientation. In order to provide better correlation between good poses and good scores, the GLIDE XP precision was favored over the standard mode.

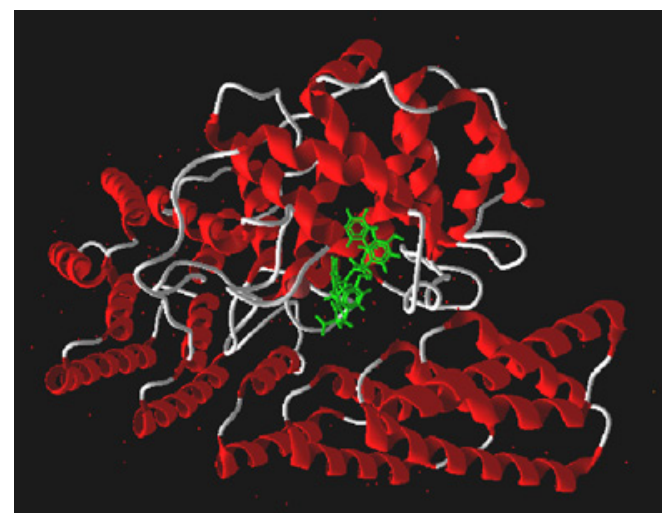

Figure 2: X-ray crystal structure of protein geranyl transferase in complex with a peptidomimetic inhibitor compound 6 (PDB ID: 3 HXB).

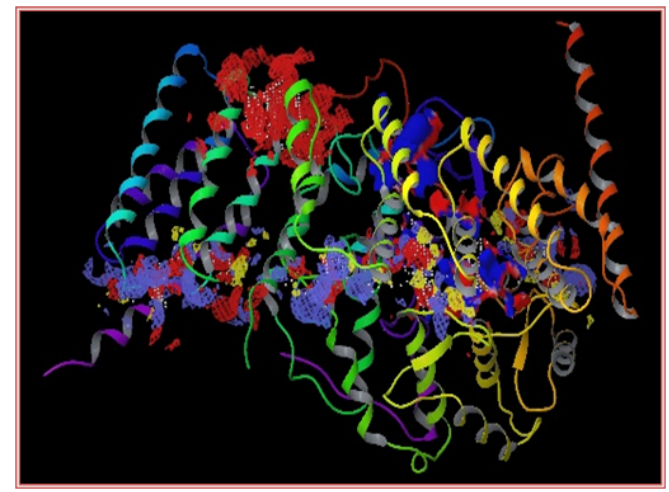

Figure 3: Sitemap image showing active site on the protein.

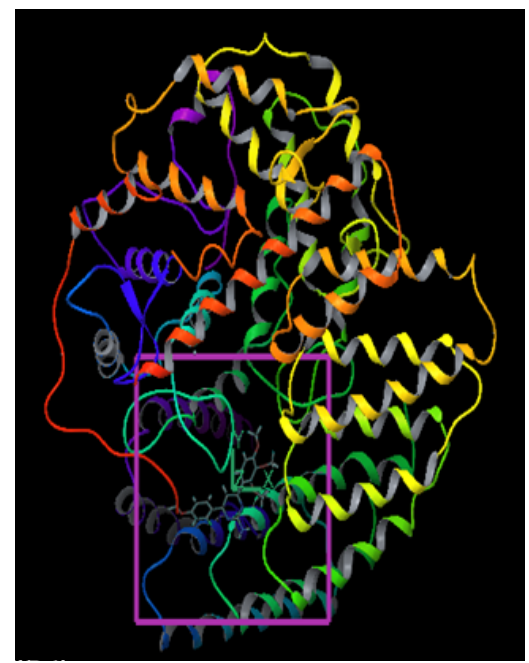

Figure 4: Receptor grid, generated to determine the size of the active site.

\section{Validation of the docking protocol}

The docking analysis was done for the ligands such as 4-(2-fluoro4-methoxyphenyl)-6-(2, 3, trimethoxyphenyl) pyrimidin-2-amine, 4-(2-fluoro-4-methoxyphenyl)-6-(2, 4, 6-trimethoxyphenyl) pyrimidin-2-amine, 4-(2-fluoro-4-methoxyphenyl)-6-(2, 4-dimethoxyphenyl) pyrimidin-2-amine, 4-(2-fluoro-4-methoxyphenyl)-6-(2,4,5-trimethoxyphenyl) pyrimidin-2-amine, 4-(2-fluoro-4-methoxyphenyl)6-(3,4,5-trimethoxyphenyl) pyrimidin-2-amine, 4-(2-fluoro-4methoxyphenyl)-6-(3,5-dimethoxyphenyl) pyrimidin-2-amine with the target protein RabGGTase using the docking software GLIDE and the docked images are shown (Figures 5-10). The Glide XP Visualizer results are given in the table 2 . All the ligands accepted the poses with the receptor RabGGTase. The structures docked by GLIDE are generally ranked according to the GLIDE Scoring Function (more negative). The scoring function of GLIDE docking program is presented in the G-score form.

The most straightforward method of evaluating the accuracy of a docking procedure is to determine how closely the lowest energy pose (binding conformation) predicted by the object scoring function. In the present study, Extra Precision GLIDE docking procedure was validated by removing the peptidomimetic inhibitor compound 6

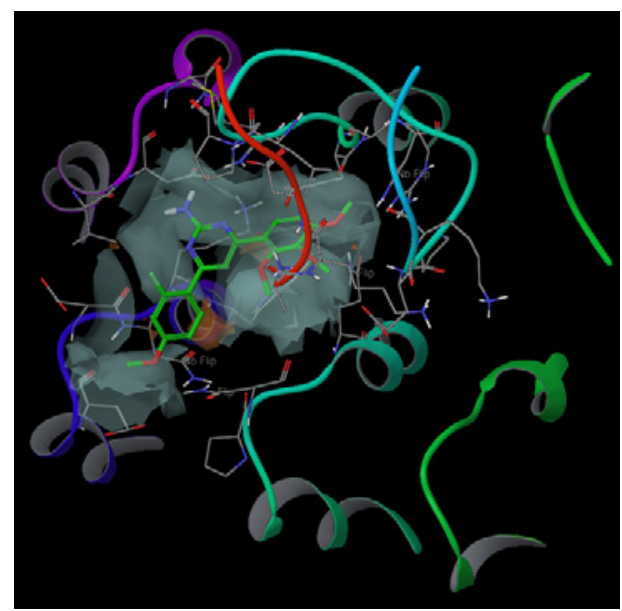

Figure 5: GLIDE docking image of ligand 1 and 3HXB.

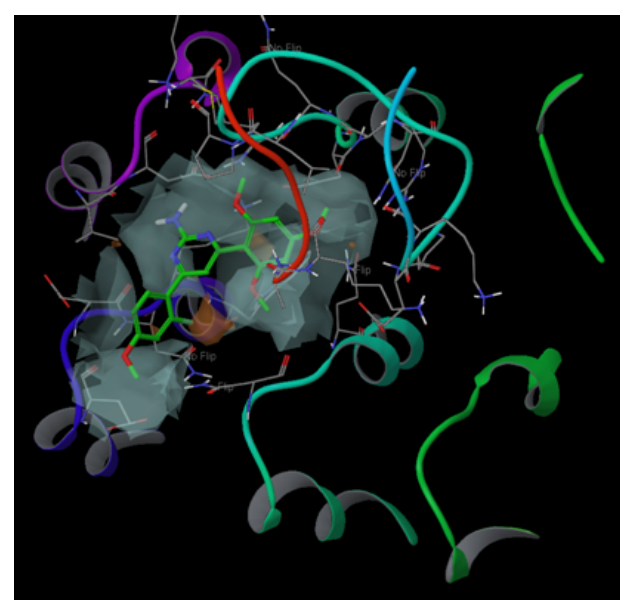

Figure 6: GLIDE docking image of ligand 2 and 3HXB. 
Citation: Ethiraj KR, Vino S, Nawas Khan F, Sajitha Lulu S (2013) Docking Studies of Pyrimidine Analogues on Geranyl Transferase. 2: 610 doi:10.4172/scientificreports. 610

Page 4 of 5

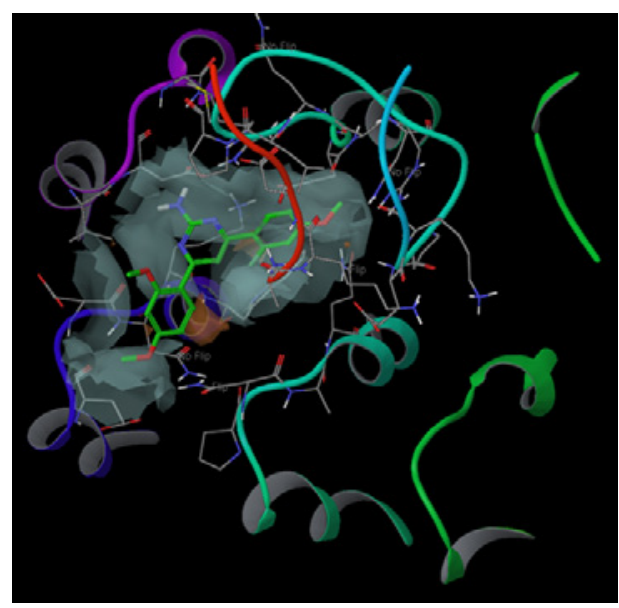

Figure 7: GLIDE docking image of ligand 3 and $3 \mathrm{HXB}$.

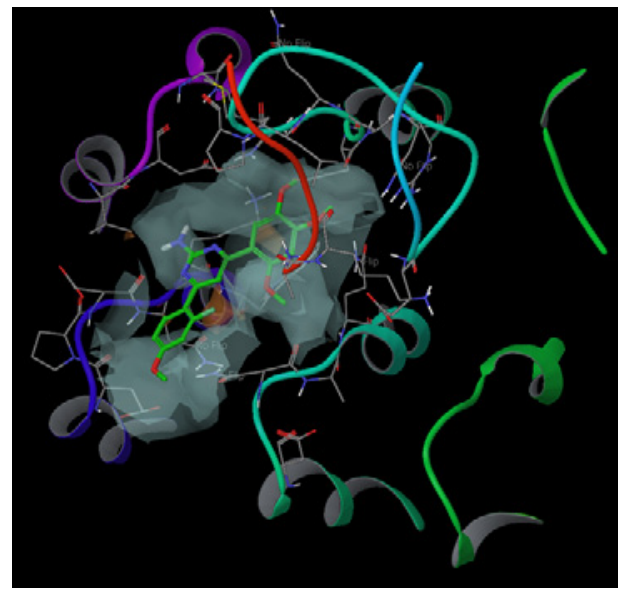

Figure 8: GLIDE docking image of ligand 4 and 3HXB.

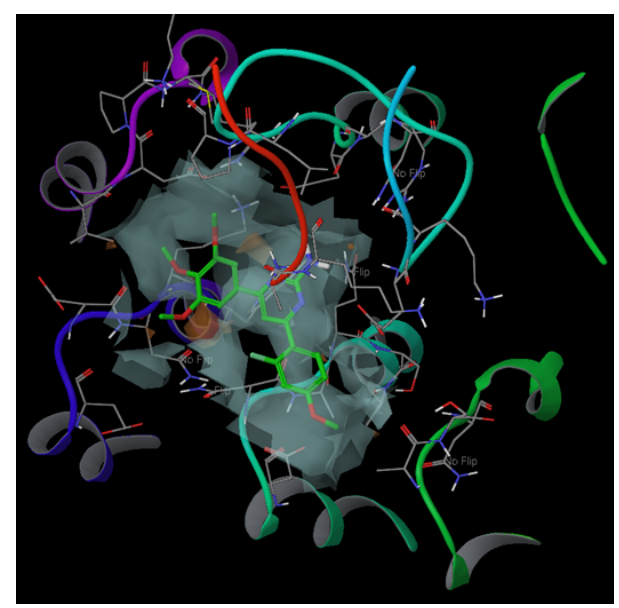

Figure 9: GLIDE docking image of ligand 5 and 3HXB.

from the binding site and redocking the six pyrimidines analogues into the binding site of RabGGTase. We found a very good agreement between the localization of the inhibitor upon docking and from the crystal structure of the protein. The binding affinity of the ligands with RabGGTase protein has been analyzed from the G-score, GLIDE energy and H-bonds. To study the molecular basis of interaction and affinity of binding of pyrimidine analogues to RabGGTase protein, all the ligands were docked into the active site of RabGGTase. The docking result of these ligands is given in table 2. The ranking of ligands was based on the GLIDE score. All the 6 ligands accepted poses with the receptor $(3 \mathrm{HXB})$. The interaction energy includes van der Waals energy, electrostatic energy, as well as intermolecular hydrogen bondings were calculated for each minimized complex (Table 2). The difference in GLIDE score among all the 6 ligands was also very small. The docking score using GLIDE varied from -1.21 to -2.3 . The GLIDE Score for Zarnestra, a standard inhibitor of farnesyltransferase, docked with RabGGTase was -3.71 . This proves that pyrimidine analogues could be potential drugs for anticancer drug development.

\section{Conclusion}

All the novel pyrimidines analogues synthesized were docked successfully on RabGGTase receptor. The GLIDE score can be used as a semi-quantitative descriptor for the ability of ligands to bind to a specific conformation of the protein receptor. Generally speaking, for low GLIDE score, good ligand affinity to the receptor may be expected. 4-(2-fluoro-4-methoxyphenyl)-6-(3,4,5-trimethoxyphenyl) pyrimidin-2-amine showed the best the inhibition for the RabGGTase receptor according to the GLIDE score obtained. The poor interaction was observed with 4-(2-fluoro-4-methoxyphenyl)-6-(3,5dimethoxyphenyl)pyrimidin-2-amine, and the order of inhibition for other analogues were, 4-(2-fluoro-4-methoxyphenyl)-6-(2,

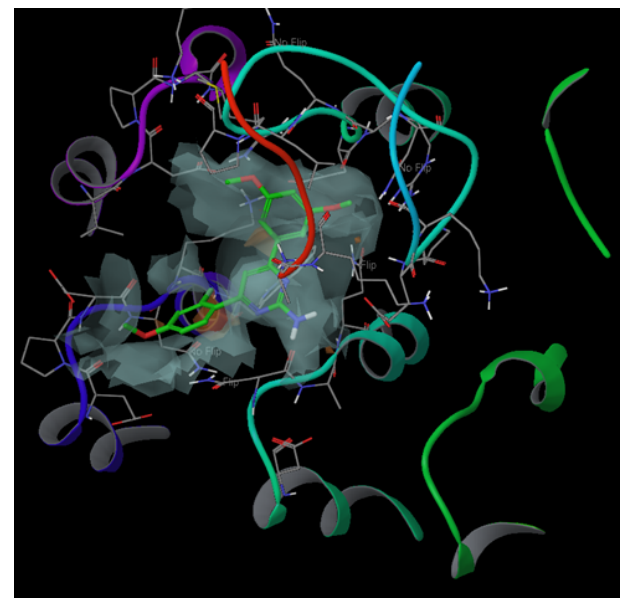

Figure 10: GLIDE docking image of ligand 6 and 3HXB

\begin{tabular}{|l|c|c|c|c|c|c|c|}
\hline Ligand & G-Score & $\begin{array}{c}\text { Lipo- } \\
\text { philic } \\
\text { EvdW }\end{array}$ & H-Bond & $\begin{array}{c}\text { Electro- } \\
\text { static } \\
\text { energy }\end{array}$ & Sitemap & $\begin{array}{c}\text { Phobic } \\
\text { Penal }\end{array}$ & $\begin{array}{c}\text { Rot } \\
\text { Penal }\end{array}$ \\
\hline $\begin{array}{c}\text { Zarnes- } \\
\text { tra }\end{array}$ & -3.71 & -1.09 & -1.25 & -2.42 & 0 & 1.15 & 0.19 \\
\hline Ligand 5 & -2.3 & -1.82 & -0.75 & -0.34 & 0 & 0.72 & 0.11 \\
\hline Ligand 2 & -2.02 & -2.32 & -1.2 & -0.28 & -0.25 & 0.14 & 0.11 \\
\hline Ligand 1 & -1.72 & -2.67 & -1.37 & -0.57 & 0 & 0 & 0.11 \\
\hline Ligand 4 & -1.55 & -1.84 & -0.79 & -0.42 & 0 & 0.47 & 0.11 \\
\hline Ligand 3 & -1.29 & -2.67 & -1.02 & -0.49 & 0 & 0.08 & 0.13 \\
\hline Ligand 6 & -1.21 & -1.98 & -0.92 & -0.18 & -0.17 & 0.23 & 0.13 \\
\hline
\end{tabular}

aThe energy data are written according to the structure generated by the docking program. All energies are given in $\mathrm{Kcal} / \mathrm{mol}$.

Table 2: Van der Waals, electrostatic, H-bond, site energy (site), G-score and penalties after GLIDE dockinga. 
Citation: Ethiraj KR, Vino S, Nawas Khan F, Sajitha Lulu S (2013) Docking Studies of Pyrimidine Analogues on Geranyl Transferase. 2: 610 doi:10.4172/scientificreports. 610

Page 5 of 5

4, 6-trimethoxyphenyl) pyrimidin-2-amine $>4$-(2-fluoro-4methoxyphenyl)-6-(2,3, trimethoxyphenyl) pyrimidin-2-amine $>4$ (2-fluoro-4-methoxyphenyl)-6-(2,4,5-trimethoxyphenyl) pyrimidin2-amine> 4-(2-fluoro-4-methoxyphenyl)-6-(2, 4-dimethoxyphenyl) pyrimidin-2-amine. Conformational analysis of different docked complexes also shows that residues Tyr 178, Ser 227 and Phe 230 play important role in this receptor's activity. Docking studies performed by GLIDE has confirmed that above inhibitors fit into the binding pocket of the RabGGTase receptor. From the results, we may observe that for successful docking, intermolecular hydrogen bonding and liphophilic interactions between the ligand and the receptor are very important. We can also explain why the GLIDE score results for 4-(2-fluoro-4methoxyphenyl)-6-(3,4,5-trimethoxyphenyl) pyrimidin-2-amine are higher than others. The main reason for the increase in GLIDE score is due to the penalties for close intra-ligand contacts.

\section{References}

1. Okamoto CT, Forte JG (2001) Vesicular trafficking machinery, the actin cytoskeleton, and $\mathrm{H}+-\mathrm{K}+-\mathrm{ATP}$ ase recycling in the gastric parietal cell. J Physiol 532: 287-296.

2. Peter M, Chavrier P, Nigg EA, Zerial M (1992) Isoprenylation of rab proteins on structurally distinct cysteine motifs. J Cell Sci 102: 857-865

3. Casey PJ, Seabra MC (1996) Protein prenyltransferases. J Biol Chem 271: 5289-5292.

4. Mattew J, Subba Rao AV, Rambhav S (1984) Propterol, an antibacterial agent from Pterocarpus marsupium. Curr Sci 53: 576-577.

5. Yamakawa T, Kagechika H, Kawachi E, Hashimoto Y, Shudo K (1990) Retinobenzoic acids. 5. Retinoidal activities of compounds having a trimethylsilyl or trimethylgermyl group(s) in human promyelocytic leukemia cells HL-60. J Med Chem 33: 1430-1437.

6. Bhat AK, Bhamana RP, Patel MR, Bellare RA, Deliwala CV (1972) Chemotherapy of fungus infections. III. Alkyl or aryl thiosemicarbazones, acid hydrazones, and styryl aryl ketones of 5-bromo- and 5-nitrosalicylaldehydes. Indian J Chem 10: 694-698.

7. Ishitsuka H, Ninomiya YT, Ohsawa C, Fujiu M, Suhara Y (1982) Direct and specific inactivation of rhinovirus by chalcone Ro 09-0410. Antimicrob Agents Chemother 22: 617-621.

8. Ninomiya $\mathrm{Y}$, Shimma N, Ishitsuka H (1990) Comparative studies on the antirhinovirus activity and the mode of action of the rhinovirus capsid binding agents, chalcone amides. Antiviral Res 13: 61-74.

9. Trivedi AR, Dodiya DK, Ravat NR, Shah VH (2008) Synthesis and biological evaluation of some new pyrimidines via a novel chalcone series. ARKIVOC 131-141.

10. Rahaman SkA, Rajendra Pasad Y, Phani Kumar, Bharath Kumar (2009) Synthesis and anti-histaminic activity of some novel pyrimidines. Saudi Pharmaceutical Journal 17: 255-258.

11. Jorgensen WL (2004) The many roles of computation in drug discovery. Science 303: 1813-1818.

12. Halgren TA, Murphy RB, Friesner RA, Beard HS, Frye LL, et al. (2004) Glide: a new approach for rapid, accurate docking and scoring. 2. Enrichment factors in database screening. J Med Chem 47: 1750-1759.

13. McRobb FM, Capuano B, Crosby IT, Chalmers DK, Yuriev E (2010) Homology modeling and docking evaluation of aminergic $G$ protein-coupled receptors. J Chem Inf Model 50: 626-637.

14. Kaminski GA, Friesner RA (2001) Evaluation and Reparametrization of the OPLS-AA Force Field for Proteins via Comparison with Accurate Quantum Chemical Calculations on Peptides. J Phys Chem B 105: 6474-6487.

15. Barreiro G, Guimarães CR, Tubert-Brohman I, Lyons TM, Tirado-Rives J, et al. (2007) Search for non-nucleoside inhibitors of HIV-1 reverse transcriptase using chemical similarity, molecular docking, and MM-GB/SA scoring. J Chem Inf Model 47: 2416-2428.

16. Dzierba CD, Tebben AJ, Wilde RG, Takvorian AG, Rafalski M, et al. (2007) Dihydropyridopyrazinones and dihydropteridinones as corticotropin-releasing factor-1 receptor antagonists: structure-activity relationships and computational modeling. J Med Chem 50: 2269-2272.

17. Halgren TA (2009) Identifying and characterizing binding sites and assessing druggability. J Chem Inf Model 49: 377-389. 